\title{
The reaction of $o$-phenylenediamine with ethoxymethylene compounds and aromatic aldehydes
}

\author{
Elina R. Marinho and Fernanda P. Proença* \\ Centro de Química, Universidade do Minho, Campos de Gualtar, 4710-057 Braga, Portugal \\ E-mail:fproenca@quimica.uminho.pt
}

\begin{abstract}
Disubstituted o-phenylenediamines 10 were generated by reaction with ethoxymethylene malononitrile, ethyl ethoxymethylene cyanoacetate and diethyl 2-(ethoxymethylene)- malonate, followed by aromatic aldehydes. These compounds proved to be highly stable even under microwave irradiation. This feature, associated to a convenient substitution pattern, makes them suitable candidates to be tested for their biological activity or to be used as ligands for the preparation of metal complex catalysts.
\end{abstract}

Keywords: o-Phenylenediamine, ethoxymethylene malononitrile, ethyl ethoxymethylene cyanoacetate, diethyl 2-(ethoxymethylene)malonate, aromatic aldehydes

\section{Introduction}

The reaction of amines and aromatic aldehydes to generate imines is a well known and extensively studied method to generate these compounds. ${ }^{1}$ When $o$-phenylenediamine $\mathbf{1}$ is reacted with an aldehyde 2 , the isolation of the monosubstituted compound $\mathbf{3}$ (Scheme 1) is not an easy task as this species either cyclizes intramolecularly to generate the 2-arylbenzimidazole $5^{2-6}$ or it rapidly incorporates another aldehyde molecule leading to the diimine $4^{7}$, that directly evolves to the $N$-benzyl-2-arylbenzimidazole $6 .^{8-10}$

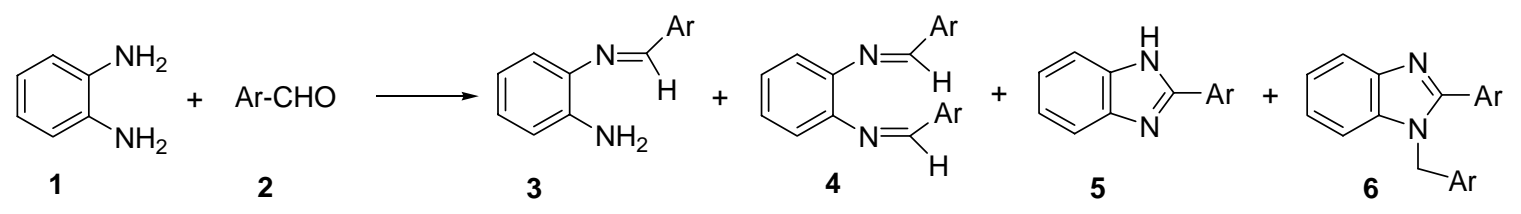

\section{Scheme 1}


The reaction of amines with ethoxymethylene compounds has also been widely used to prepare the corresponding aminomethylene derivatives. ${ }^{11,12}$

The present work is part of a project aiming at the synthesis of flexible analogs of clozapine, a golden standard for the treatment of schizophrenia, used when other medications fail due to the severe adverse side effects, in particular agranulocytosis. Synthetic efforts were centered on the use of $o$-phenylenediamine as a precursor of disubstituted compounds, where different moieties were incorporated in each aromatic amino group.

\section{Results and Discussion}

In this work, the diamine $\mathbf{1}$ was reacted with 1.2 molar equivalents of 4-hydroxybenzaldehyde and the solids were grounded in a mortar for $5 \mathrm{~min}$. The solid was suspended in water and stirred at $\mathrm{rt}$ for $1 \mathrm{~h}$ leading to product 3 (Scheme 1, $\mathrm{Ar}=4-\mathrm{HOC}_{6} \mathrm{H}_{4}$ ), isolated in 99\% yield. In DMSO$\mathrm{d}_{6}$ solution, this compound rapidly evolved to benzimidazole 5 . Considering that intramolecular cyclization was difficult to prevent, this synthetic approach was abandoned and the diamine $\mathbf{1}$ was initially reacted with ethoxymethylene compounds $\mathbf{7 a - c}{ }^{13,14}$. The reaction was carried out in ethanol and product 8 rapidly precipitates form solution as an off-white solid (Table 1) and is isolated in an analytically pure form by a simple filtration.

Table 1. Reaction of $o$-phenylenediamine with ethoxymethylene compounds

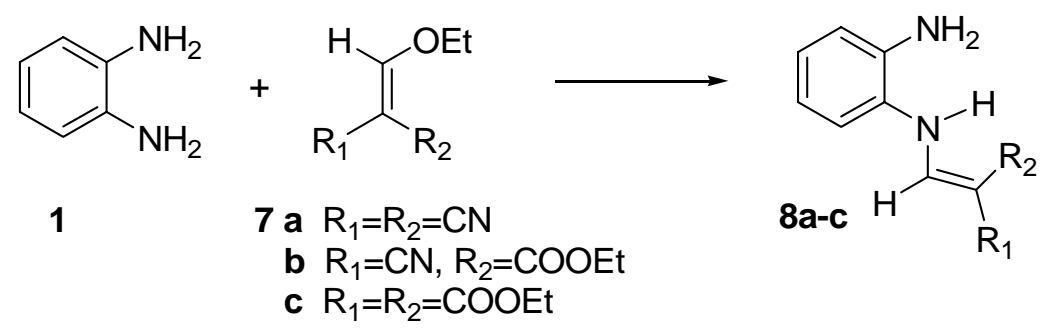

\begin{tabular}{cccc}
\hline Entry & Reactions conditions & Product (yield) & Ref. \\
\hline 1 & $\mathbf{1}+\mathbf{7 a}(1: 1)$, EtOH, rt, 5 min & $\mathbf{8 a}(56 \%)$ & 15,16 \\
2 & $\mathbf{1}+\mathbf{7 b}(1: 1)$, EtOH, rt, 5 min & $\mathbf{8 b}(89 \%)$ & 17 \\
3 & $\mathbf{1}+\mathbf{7 c}(1: 1)$, EtOH, rt, $\mathrm{h}$ & $\mathbf{8 c}(89 \%)$ & \\
\hline
\end{tabular}

The lower isolated yield of compound 8a is probably due to a facile intramolecular cyclization to generate benzimidazole 9 (Scheme 2) with elimination of malononitrile. This evolution can be clearly followed by ${ }^{1} \mathrm{H}$ NMR of a solution of 8a $(15 \mathrm{mg})$ in DMSO-d 6 (650 $\left.\mu \mathrm{L}\right)$. Compound 9 (16\%) was already present in the freshly prepared solution and after 6 days at $\mathrm{rt}$ complete evolution occurred. The structure of compound $\mathbf{9}$ was confirmed by elemental analysis, IR and NMR spectroscopy, including HMBC and HMQC correlation spectra. 


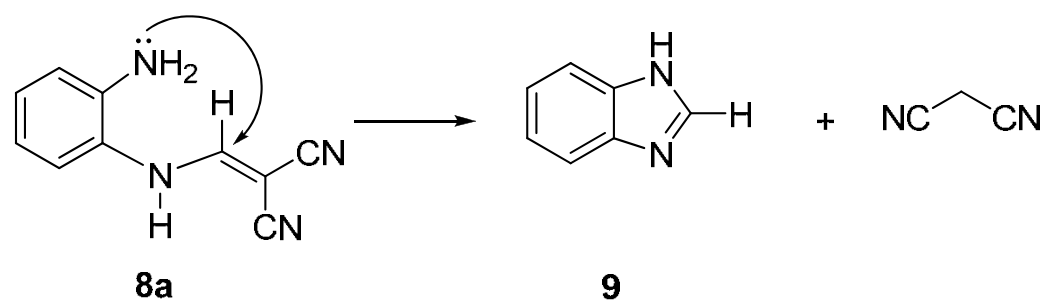

Scheme 2. Evolution of compound 8a in DMSO- $\mathrm{d}_{6}$ solution.

Compound 8 was also fully characterized by elemental analysis and spectroscopic techniques, including NMR correlation techniques, allowing an unequivocal band assignment. Two sets of bands were identified in the ${ }^{1} \mathrm{H}$ and ${ }^{13} \mathrm{C}$ spectra of compounds $\mathbf{8 a}$ and $\mathbf{8 b}$, assigned to different isomers A and B. An important observation is that for one of the isomers (identified as A), coupling is observed between the $\mathrm{N}-\mathrm{H}$ and $\mathrm{C}-\mathrm{H}$ protons of the enamine moiety $(\mathbf{8 a}, \delta \mathrm{NH}$ $10.20, \delta \mathrm{CH} 7.72, \mathrm{~d}, J=7.2 \mathrm{~Hz} ; \mathbf{8 b}, \delta \mathrm{NH} 10.50, \delta \mathrm{CH} 8.31, \mathrm{~d}, J=13.6 \mathrm{~Hz})$. For isomer B, these two signals are broad singlets, possibly as a result of an unresolved small coupling constant $(\mathbf{8 a}, \delta$ NH $9-11, \delta$ CH $8.04 ; 8$ bb $\delta$ NH 9.95, $\delta$ CH 7.94). The Karplus equation, ${ }^{18}$ has been extensively used as an indication of dihedral angles, from their correlation with vicinal coupling constants. Small magnitude coupling constants are expected as the result of dihedral angles close to $90{ }^{\circ} \mathrm{C}$, while large values for the coupling constant correspond to angles of $0{ }^{\circ} \mathrm{C}$ and $180{ }^{\circ} \mathrm{C}$. For compounds 8, this was rationalized by the presence of two different intramolecular H-bonds involving the aromatic amino groups, as is represented in Figure 1.<smiles>N#CC(C#N)=CNc1ccccc1N</smiles>

8a<smiles>CCOC(=O)/C(C#N)=C\Nc1ccccc1N</smiles>

$8 b$

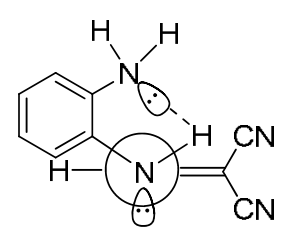

8 a (A) $22 \%$

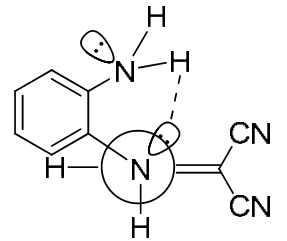

8a (B) $78 \%$

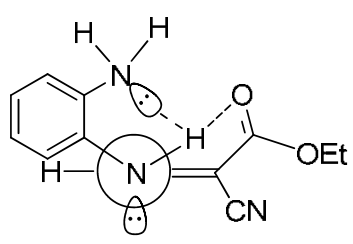

$8 b$ (A) $55 \%$

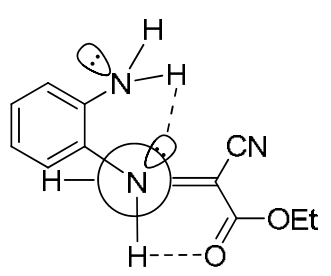

8 b (B) $\quad 45 \%$

Figure 1. Newman projection of isomers A and B for compounds $\mathbf{8 a}$ and $\mathbf{8 b}$.

The presence of two conformers was previously reported in the literature for compounds analogous to 8a and $\mathbf{8 b}$. Studies by IR and NMR spectroscopy and ab initio calculations support these observations for (phenylamino)methylene propanedinitrile ${ }^{19}$ and for other push-pull enamines with two cyano substituents or one cyano and one acetyl group ${ }^{20}$. The presence of an 
intramolecular hydrogen bond was recognized as an important influence on the conformational behaviour of this type of molecules ${ }^{21}$. The IR, Raman and NMR spectra of 2-aminomethylene2,4-pentanedione indicate that this compound exists as a single isomer ${ }^{22}$ and this was also the case for the analogous structure 8c, prepared and fully characterized in this work.

The reaction of $o$-phenylenediamine with ethoxymethylene cyanoacetate was also carried out under microwave irradiation, in the absence and in the presence of base (DBU). The results, summarized in Table 2, indicate that the condensation product $\mathbf{8 b}$ is quantitatively formed when a 1:1 molar ratio of the reagents is irradiated at $300 \mathrm{~W}$ for $3 \mathrm{~min}$ (Table 2, entry 1). Addition of 1 equivalent of DBU followed by $7 \mathrm{~min}$ under $300 \mathrm{~W}$ lead to the benzimidazole 9 as the only product (Table 2, entry 2).

When a catalytic amount of DBU was used and the mixture was irradiated at $300 \mathrm{~W}$ for $4 \mathrm{~min}$, a 2:1 mixture of the condensation product $\mathbf{8 b}$ and of $o$-phenylenediamine $\mathbf{1}$ were identified by ${ }^{1} \mathrm{H}$ NMR. Traces of the cyclic benzimidazole $\mathbf{9}$ can also be detected (Table 2, entry 3 ).

Table 2. Reaction of $o$-phenylenediamine $1(0.13 \mathrm{~g})$ with ethyl ethoxymethylene cyanoacetate $(0.20 \mathrm{~g})$ under microwave conditions

\begin{tabular}{ccc}
\hline Entry & Reactions conditions & Products $^{\text {a }}$ \\
\hline 1 & neat reagents, 300 W, 3 min & $\mathbf{8 b}$ \\
2 & neat reagents, DBU (1 equiv. $)$ & $\mathbf{9}$ \\
& $300 \mathrm{~W}, 7$ min & \\
3 & neat reagents, DBU (cat.) & $\mathbf{1}+\mathbf{8 b}(1: 2$ ratio $){ }^{\mathrm{b}}$ \\
& $300 \mathrm{~W}, 4$ min &
\end{tabular}

${ }^{a}$ by ${ }^{1} \mathrm{H}$ NMR. ${ }^{b}$ traces of 9 were also detected in the ${ }^{1} \mathrm{H}$ NMR spectrum.

Microwave radiation was used to induce intramolecular cyclization on compounds $\mathbf{8 a}, \mathbf{8 b}$ and 8c. The success of this reaction was expected to depend on the formation of the appropriate conformational isomer. Microwave would assist the interconversion of conformers through an efficient bond rotation and was expected to generate the benzodiazepine ring through a favoured 7-exo-dig cyclization process (Scheme 3, pathway a)). ${ }^{16,23,24}$

The reaction was initially performed on a kitchen microwave oven at the $800 \mathrm{~W}$ power level. The solid samples (8a, $12.3 \mathrm{mg} ; \mathbf{8 b}, 12.6 \mathrm{mg}$ and $\mathbf{8 c}, 14.4 \mathrm{mg}$ ) were irradiated for $1 \mathrm{~min}$, followed by an extra period of 2 minutes and then 5 minutes. After a total of 8 minutes of radiation, no evolution was detected by tlc (Table 3, entries 1,3,5). The samples were irradiated for a further 30 minutes, and the compounds were analysed by ${ }^{1} \mathrm{H}$ NMR. Compounds $\mathbf{8 b}$ and $\mathbf{8 c}$ were recovered unchanged (Table 3, entries 4 and 6) but 8a was a 2:1 mixture of benzimidazole 9 and starting material $\mathbf{8 a}$ (Table 3, entry 2). 


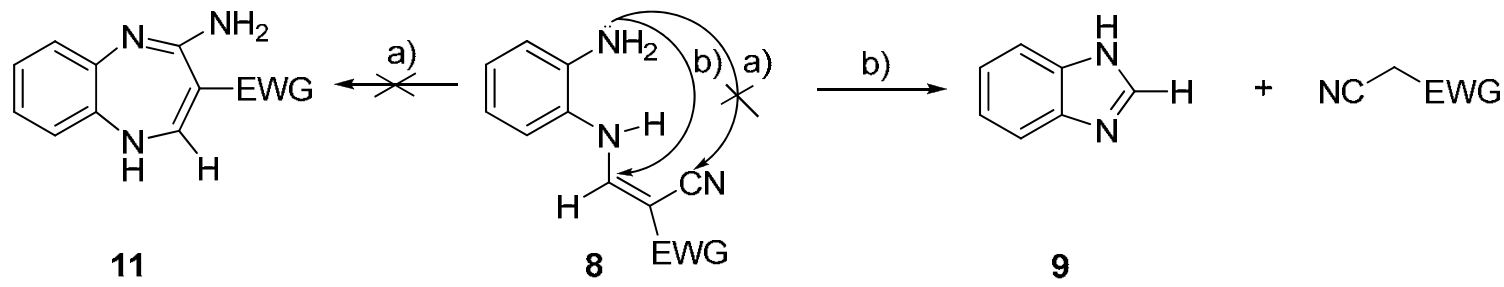

Scheme 3. Possible pathways [a) or b)] for the intramolecular cyclization of compounds 8.

In this case, a 5-exo-dig intramolecular cyclization occurred to generate the imidazole ring (not the 7-membered ring) with elimination of malononitrile, also identified in the ${ }^{1} \mathrm{H}$ NMR spectrum (Scheme 3).

A new set of experiments were performed using the scientific microwave equipment and compounds $\mathbf{8 b}(8.6 \mathrm{mg})$ and $\mathbf{8 c}(9.0 \mathrm{mg})$ were irradiated at a constant power of $300 \mathrm{~W}$ for $14 \mathrm{~min}$ and than at $600 \mathrm{~W}$ for 1 hour. In both cases, no evolution was detected by tlc (Table 3, entries 7 and 10). $N$-Methylpiperazine (0.5 molar equivalents) was added and the compounds were irradiated at $300 \mathrm{~W}$. After a total of 32 minutes, compound $\mathbf{8 b}$ led to a homogeneous solution and ${ }^{1} \mathrm{H}$ NMR indicated that intramolecular cyclization was the major pathway leading to benzimidazole 9. (Table 3, entry 8).

Table 3. Evolution of compounds $\mathbf{8 a}, \mathbf{8 b}$ and $\mathbf{8 c}$ under microwave irradiation

\begin{tabular}{cccc}
\hline Entry & Compound & Exp. conditions & Product \\
\hline 1 & $\mathbf{8 a}(12.3 \mathrm{mg})$ & $800 \mathrm{~W}, 8 \mathrm{~min}$ & $\mathbf{8 a}^{\mathrm{a}}$ \\
2 & & $800 \mathrm{~W}, 38 \mathrm{~min}$ & $\mathbf{8 a}+\mathbf{9}(1: 2 \text { ratio })^{\mathrm{b}}$ \\
3 & $\mathbf{8 b}(12.6 \mathrm{mg})$ & $800 \mathrm{~W}, 8 \mathrm{~min}$ & $\mathbf{8 b}^{\mathrm{a}}$ \\
4 & & $800 \mathrm{~W}, 38 \mathrm{~min}$ & $\mathbf{8 b}^{\mathrm{b}}$ \\
5 & $\mathbf{8 c}(14.4 \mathrm{mg})$ & $800 \mathrm{~W}, 8 \mathrm{~min}$ & $\mathbf{8 c}^{\mathrm{a}}$ \\
6 & & $800 \mathrm{~W}, 38 \mathrm{~min}$ & $\mathbf{8 c}^{\mathrm{b}}$ \\
7 & $\mathbf{8 b}(8.6 \mathrm{mg})$ & i) $300 \mathrm{~W}, 14 \mathrm{~min}$ & $\mathbf{8 b}^{\mathrm{a}}$ \\
& & ii) $600 \mathrm{~W}, 1 \mathrm{~h}$ & $\mathbf{9}^{\mathrm{c}}$ \\
8 & & $N$-methylpiperazine $(0.5 \mathrm{equiv})$ & $\mathbf{9}^{\mathrm{c}}$ \\
9 & $\mathbf{8 b}(37.4 \mathrm{mg})$ & $\mathrm{DMSO}(0.2 \mathrm{~mL}), 600 \mathrm{~W}, 1 \mathrm{~h}$ & $\mathbf{8 c}^{\mathrm{a}}$ \\
10 & $\mathbf{8 c}(9.0 \mathrm{mg})$ & i) $300 \mathrm{~W}, 14 \mathrm{~min}$ & \\
& & ii) $600 \mathrm{~W}, 1 \mathrm{~h}$ & $\mathbf{8 c}^{\mathrm{b}}$ \\
11 & & $N-$ methylpiperazine $(0.5 \mathrm{equiv})$ & $\mathbf{8 c}^{\mathrm{b}}$ \\
\hline
\end{tabular}

${ }^{\mathrm{a}}$ by tlc. ${ }^{\mathrm{b}}$ by ${ }^{1} \mathrm{H}$ NMR. ${ }^{\mathrm{c}}$ quantitative yield, by ${ }^{1} \mathrm{H}$ NMR. 
Addition of DMSO to compound $\mathbf{8 b}$ followed by 1 hour at $600 \mathrm{~W}$ (Table 3, entry 9) also resulted in the formation of benzimidazole 9 (an equimolar amount of ethyl 2-cyanoacetate was equally identified in the ${ }^{1} \mathrm{H}$ NMR spectrum).

Compound 8c (Table 3, entry 11) remained unchanged after $32 \mathrm{~min}$ at $300 \mathrm{~W}$. An aqueous solution of sodium carbonate $(2 \mathrm{M}, 1 \mathrm{~mL})$ was added to this compound and the mixture was than irradiated for $1 \mathrm{~min}$ at $300 \mathrm{~W}$. The solid obtained after complete removal of the solvent was identified by ${ }^{1} \mathrm{H}$ NMR as $8 \mathrm{c}$ (Table 3 , entry 12 ).

The unsuccess to generate a 7-membered ring by intramolecular cyclization of compounds 8a, $\mathbf{8 b}$ and $\mathbf{8 c}$, led us to prepare compounds 10, from the reaction of $\mathbf{8}$ with aromatic aldehydes $\mathbf{2}$ (Table 4) and to study their reactivity under microwave conditions.

The best experimental conditions for the reaction of substituted aniline $\mathbf{8 b}$ with aldehydes $\mathbf{2}$ corresponds to the use of a 1:1 molar ratio of these reagents in acetonitrile and in the presence of 1 drop of piperidine. The product precipitates form the reaction medium as a yellow solid and is isolated in good yield (Table 4, entries 2-7).

Table 4. Reaction of compounds 8a-c with aromatic aldehydes<smiles>[R]C([R])=CNc1ccccc1N</smiles>

8a, $R_{1}=R_{2}=C N$

b, $\mathrm{R}_{1}=\mathrm{CN}, \mathrm{R}_{2}=\mathrm{COOEt}$

c, $R_{1}=R_{2}=$ COOEt
$\mathrm{Ar}-\mathrm{CHO}$

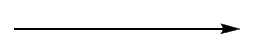

2a, $\mathrm{Ar}=4-\mathrm{HOC}_{6} \mathrm{H}_{4}$

b, $\mathrm{Ar}=2-\mathrm{HO}-3-\mathrm{MeOC}_{6} \mathrm{H}_{3}$

c, $\mathrm{Ar}=4-\mathrm{MeOC}_{6} \mathrm{H}_{4}$

d, $\mathrm{Ar}=4-\mathrm{ClC}_{6} \mathrm{H} 4$

e, $\mathrm{Ar}=4-\mathrm{NCC}_{6} \mathrm{H}_{4}$

f, $\mathrm{Ar}=4-\mathrm{O}_{2} \mathrm{NC}_{6} \mathrm{H}_{4}$<smiles>[R]C([R])=CN([2H])c1ccccc1/N=C/Br</smiles>

10a, $\mathrm{R}_{1}=\mathrm{R}_{2}=\mathrm{CN}, \mathrm{Ar}=2-\mathrm{HO}-3-\mathrm{MeOC}_{6} \mathrm{H}_{3}$

b, $\mathrm{R}_{1}=\mathrm{CN}, \mathrm{R}_{2}=\mathrm{cOOEt}, \mathrm{Ar}=4-\mathrm{HOC}_{6} \mathrm{H}_{4}$

c, $\mathrm{R}_{1}=\mathrm{CN}, \mathrm{R}_{2}=\mathrm{COOEt}, \mathrm{Ar}=4-\mathrm{MeOC}_{6} \mathrm{H}_{4}$

d, $\mathrm{R}_{1}=\mathrm{CN}, \mathrm{R}_{2}=\mathrm{COOEt}, \mathrm{Ar}=4-\mathrm{ClC}_{6} \mathrm{H}_{4}$

e, $\mathrm{R}_{1}=\mathrm{CN}, \mathrm{R}_{2}=\mathrm{COOEt}, \mathrm{Ar}=4-\mathrm{NCC}_{6} \mathrm{H}_{4}$

f, $\mathrm{R}_{1}=\mathrm{CN}, \mathrm{R}_{2}=\mathrm{COOEt}, \mathrm{Ar}=4-\mathrm{O}_{2} \mathrm{NC}_{6} \mathrm{H}_{4}$

g, $\mathrm{R}_{1}=\mathrm{CN}, \mathrm{R}_{2}=\mathrm{COOEt}, \mathrm{Ar}=2-\mathrm{HO}-3-\mathrm{MeOC}_{6} \mathrm{H}_{3}$

h, $\mathrm{R}_{1}=\mathrm{R}_{2}=\mathrm{COOEt}, \mathrm{Ar}=4-\mathrm{HOC}_{6} \mathrm{H}_{4}$

i, $\mathrm{R}_{1}=\mathrm{R}_{2}=$ COOEt, $\mathrm{Ar}=4-\mathrm{MeOC}_{6} \mathrm{H}_{4}$

j, $\mathrm{R}_{1}=\mathrm{R}_{2}=$ COOEt, $\mathrm{Ar}=4-\mathrm{ClC}_{6} \mathrm{H}_{4}$

$\mathbf{k}, \mathrm{R}_{1}=\mathrm{R}_{2}=$ COOEt, $\mathrm{Ar}=4-\mathrm{NCC}_{6} \mathrm{H}_{4}$

I, $\mathrm{R}_{1}=\mathrm{R}_{2}=$ COOEt, $\mathrm{Ar}=4-\mathrm{O}_{2} \mathrm{NC}_{6} \mathrm{H}_{4}$

m, $\mathrm{R}_{1}=\mathrm{R}_{2}=\mathrm{COOEt}, \mathrm{Ar}=2-\mathrm{HO}-3-\mathrm{MeOC}_{6} \mathrm{H}_{3}$

\begin{tabular}{ccc}
\hline Entry & Reaction conditions $^{\text {a }}$ & Product (yield) \\
\hline 1 & $\mathbf{8 a}+\mathbf{2 b}, \mathrm{rt}, 25 \mathrm{~min}$ & $\mathbf{1 0 a}(48 \%)$ \\
2 & $\mathbf{8 b}+\mathbf{2 a}, \mathrm{rt}, 1.5 \mathrm{~h}$ & $\mathbf{1 0 b}(95 \%)$ \\
3 & $\mathbf{8 b}+\mathbf{2 c}, \mathrm{rt}, 10 \mathrm{~min}$ & $\mathbf{1 0 c}(83 \%)$ \\
4 & $\mathbf{8 b}+\mathbf{2 d}, \mathrm{rt}, 15 \mathrm{~min}$ & $\mathbf{1 0 d}(81 \%)$ \\
5 & $\mathbf{8 b}+\mathbf{2 e}, \mathrm{rt}, 10 \mathrm{~min}$ & $\mathbf{1 0 e}(89 \%)$ \\
6 & $\mathbf{8 b}+\mathbf{2 f}, \mathrm{rt}, 5 \mathrm{~min}$ & $\mathbf{1 0 f}(86 \%)$ \\
7 & $\mathbf{8 b}+\mathbf{2 b}, \mathrm{rt}, 1$ day & $\mathbf{1 0 g}(80 \%)$ \\
\hline
\end{tabular}


Table 4. Continued

\begin{tabular}{ccc}
\hline Entry & Reaction conditions $^{\mathrm{a}}$ & Product (yield) \\
\hline 8 & $\mathbf{8 c}+\mathbf{2 a}, \mathrm{rt}, 5 \mathrm{~h}, 10^{\circ} \mathrm{C}, 3$ days & $\mathbf{1 0 h}(55 \%)$ \\
9 & $\mathbf{8 c}+\mathbf{2 c}, \mathrm{rt}, 3$ days & $\mathbf{1 0 i}(74 \%)$ \\
10 & $\mathbf{8 c}+\mathbf{2 d}, \mathrm{rt}, 5 \mathrm{~h}, 10^{\circ} \mathrm{C}, 10 \mathrm{~min}$ & $\mathbf{1 0 j}(76 \%)$ \\
11 & $\mathbf{8 c}+\mathbf{2 e}, \mathrm{rt}, 17 \mathrm{~h}$ & $\mathbf{1 0 k}(81 \%)$ \\
12 & $\mathbf{8 c}+\mathbf{2 f}, \mathrm{rt}, 2.5 \mathrm{~h}$ & $\mathbf{1 0 l}(61 \%)$ \\
13 & $\mathbf{8 c}+\mathbf{2 b}, \mathrm{rt}, 1$ day & $\mathbf{1 0 m}(93 \%)$ \\
\hline
\end{tabular}

${ }^{a}$ A 1:1 mixture of compounds 8 and 2 was reacted in acetonitrile and piperidine (1drop).

The reaction of compound $\mathbf{8 a}$ with aldehyde $\mathbf{2 b}$ under these optimized conditions (Table 4 , entry 1) led to a lower yield of product 10a (48\%). When aldehyde $2 \mathbf{a}$ was used in this reaction, the only product isolated was the corresponding arylidenemalononitrile (43\%) probably arising form the reaction of $\mathbf{2 a}$ with malononitrile, formed as a result of intramolecular cyclization of $\mathbf{8 a}$ (Scheme 2). This competitive reaction usually leads to complex mixtures, from where it is difficult to isolate a pure product and no further reactions were performed.

The reaction of 8c with aldehydes 2 led to products 10h-m (Table 4, entries 8-13) more soluble in acetonitrile than the analogous structures 10b-g. (Table 4, entries 2-7). Most of these products were isolated as two separate crops, one corresponding to the solid that precipitates directly form the reaction medium and another obtained after removal of the solvent. The values quoted in table 4 for the yield of these compounds refer to the total isolated yield.

Compounds 10a-m were fully characterized and the NMR spectrum shows that only one isomer is present in DMSO- $\mathrm{d}_{6}$ solution. Coupling was always observed between the $\mathrm{NH}$ and $\mathrm{CH}$ protons of the enamine moiety for compounds 10c-m $(\delta \mathrm{NH} 11.5-12.0 \mathrm{ppm}, \delta \mathrm{CH} 8.5-8.7 \mathrm{ppm}, J$ approximately $14 \mathrm{~Hz}$ ). The presence of a single isomer is probably the result of imine formation. This leads to one possible intramolecular H-bond involving both aromatic nitrogen atoms (Figure 2, structure 10 (A). A preferred imine configuration is foreseen for compounds 10 due to steric hindrance caused by the bulky aromatic ring.

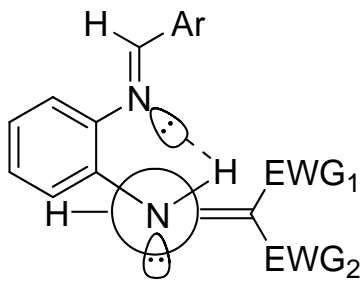

$10(A)$

Figure 2. Newman projection of isomer A for compounds 10. 
Studies were also carried out on the reactivity of compounds 10e, 10c and 10l, under microwave irradiation. In this case, the enamine moiety was expected to cyclize intramolecularly with the imine carbon atom, leading to a 7-membered ring system through a favored 7-endo-trig process. This compound could evolve to the oxidized structure $\mathbf{1 1}$ or to the diazepine $\mathbf{1 2}$ after elimination of $\mathrm{HCN}$ (when $\mathrm{EWG}_{1}=\mathrm{CN}$ ) (Scheme 4).
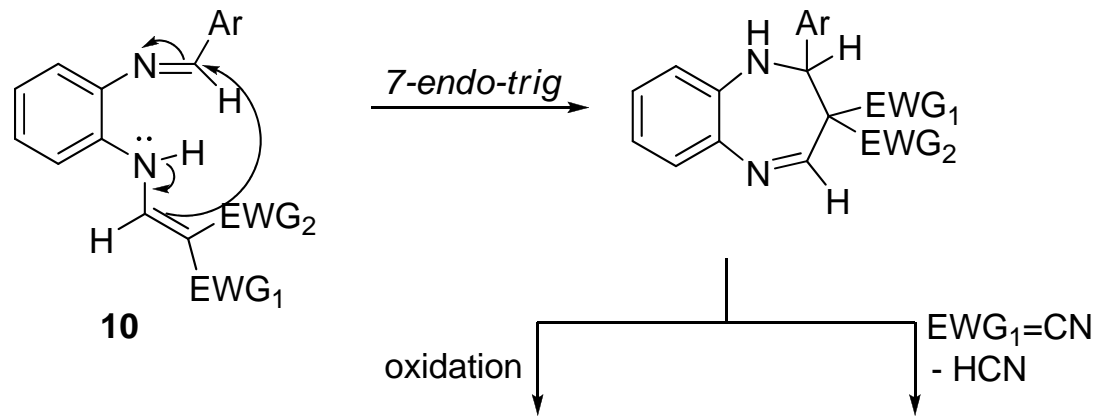

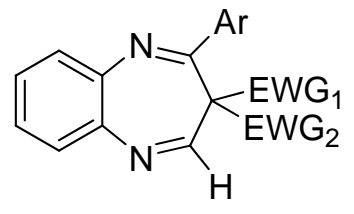

11<smiles>O=[N+]([O-])C1=C(Br)Nc2ccccc2N=C1</smiles>

12

Sheme 4. Possible evolution of compounds $\mathbf{1 0}$ under microwave conditions.

No evolution was detected by tlc on compound 10e after microwave irradiation (100 W, 1 min; $200 \mathrm{~W}, 1 \mathrm{~min} ; 400 \mathrm{~W}, 1 \mathrm{~min} ; 300 \mathrm{~W}, 7 \mathrm{~min}$ ) ( Table 5, entries 1, 2, 3).

Addition of basic alumina (Table 5, entry 4) or Montmorillonite K10 (Table 5, entry 5) followed by microwave irradiation that was raised to $600 \mathrm{~W}(1 \mathrm{~min})$ was equally unsuccessful.

Compounds 10c and 10l were also found to be stable upon irradiation at $300 \mathrm{~W}$ (14 min) and $600 \mathrm{~W}$ (60 min) (Table 5, entries 6 and 10).

Addition of $\mathrm{N}$-methylpiperazine ( 0.5 molar equivalents) followed by $52 \mathrm{~min}$ at $300 \mathrm{~W}$ (Table 5 , entries 7 and 11) or addition of $2 \mathrm{M}$ sodium carbonate $(1 \mathrm{~mL})$ with irradiation at $300 \mathrm{~W}$ for $2 \mathrm{~min}$ (Table 5, entries 8 and 12) resulted in the recovery of the starting material.

Addition of DMSO to compound 10c (Table 5, entry 9) followed by 1 hour at $300 \mathrm{~W}$ and 1 hour at $600 \mathrm{~W}$ led to a 1:1 mixture of benzimidazole 9, and the corresponding arylidene cyanoacetate (identified in the ${ }^{1} \mathrm{H}$ NMR spectrum). 
Table 5. Evolution of compounds 10e, 10c and 101 under microwave irradiation

\begin{tabular}{|c|c|c|c|}
\hline Entry & Compound & Experimental conditions & Product \\
\hline 1 & 10e $(9.6 \mathrm{mg})$ & $\begin{array}{c}100 \mathrm{~W}, 1 \mathrm{~min} ; 200 \mathrm{~W}, 1 \mathrm{~min} ; 400 \mathrm{~W}, \\
1 \mathrm{~min}\end{array}$ & $10 e^{a)}$ \\
\hline 2 & & $300 \mathrm{~W}, 2 \mathrm{~min}$ & $10 e^{a)}$ \\
\hline 3 & & $300 \mathrm{~W}, 5 \mathrm{~min}$ & $10 \mathrm{e}^{\mathrm{a})}$ \\
\hline \multirow[t]{2}{*}{4} & $\mathbf{1 0 e}(10.8 \mathrm{mg})$ & basic alumina (31.4 mg) & $10 \mathrm{e}^{\mathrm{a})}$ \\
\hline & & $\begin{array}{c}100 \mathrm{~W}, 1 \mathrm{~min} ; 200 \mathrm{~W}, 1 \mathrm{~min} ; 400 \mathrm{~W} \\
1 \mathrm{~min} ; 600 \mathrm{~W}, 1 \mathrm{~min} ; 800 \mathrm{~W}, 1 \mathrm{~min} \\
1600 \mathrm{~W}, 1 \mathrm{~min}\end{array}$ & \\
\hline \multirow[t]{2}{*}{5} & $\mathbf{1 0 e}(10.6 \mathrm{mg})$ & Montmorillonite K 10 (14 mg) & $10 e^{a)}$ \\
\hline & & $\begin{array}{c}200 \mathrm{~W}, 1 \mathrm{~min} ; 400 \mathrm{~W}, 1 \mathrm{~min} ; 800 \mathrm{~W} \\
6 \mathrm{~min} ; 1600 \mathrm{~W}, 1 \mathrm{~min}\end{array}$ & \\
\hline 6 & 10c $(53 \mathrm{mg})$ & $300 \mathrm{~W}, 14 \mathrm{~min} ; 600 \mathrm{~W}, 60 \mathrm{~min}$ & $10 c^{a)}$ \\
\hline \multirow[t]{2}{*}{7} & & $N$-methylpiperazine ( 0.5 equiv.) & $10 c^{a)}$ \\
\hline & & $300 \mathrm{~W}, 52 \mathrm{~min}$ & \\
\hline 8 & & $\mathrm{Na}_{2} \mathrm{CO}_{3} 2 \mathrm{M}(1 \mathrm{~mL}), 300 \mathrm{~W}, 2 \mathrm{~min}$ & $10 c^{b)}$ \\
\hline \multirow[t]{3}{*}{9} & 10c (34 mg) & $\operatorname{DMSO}(0.2 \mathrm{~mL})$ & \\
\hline & & i) $300 \mathrm{~W}, 1 \mathrm{~h}$ & \\
\hline & & ii) $600 \mathrm{~W}, 1 \mathrm{~h}$ & $9^{\text {b) }}$ \\
\hline 10 & 101 (36 mg) & $300 \mathrm{~W}, 14 \mathrm{~min} ; 600 \mathrm{~W}, 60 \mathrm{~min}$ & $101^{\mathrm{a})}$ \\
\hline \multirow[t]{2}{*}{11} & & $N$-methylpiperazine ( 0.5 equiv.) & $101^{\text {a) }}$ \\
\hline & & $300 \mathrm{~W}, 52 \mathrm{~min}$ & \\
\hline 12 & & $\mathrm{Na}_{2} \mathrm{CO}_{3} 2 \mathrm{M}(1 \mathrm{~mL}), 300 \mathrm{~W}, 2 \mathrm{~min}$ & $101^{\mathrm{b})}$ \\
\hline
\end{tabular}

${ }^{a}$ by tlc. ${ }^{b}$ by ${ }^{1} \mathrm{H}$ NMR.

\section{Conclusions}

In conclusion, the disubstituted o-phenylenediamine 10, generated by reaction with substituted ethoxymethylene compounds followed by aromatic aldehydes, proved to be a highly stable structure even under microwave irradiation. This behavior does not compare with that reported for mono- and disubstituted arylidene o-phenylenediamines, which are unstable and cyclize spontaneously, in solution, to the 2-arylbenzimidazole or to the $N$-benzyl-2-arylbenzimidazole.

The neat solid 10 was exposed to an increasing radiation power (form $100 \mathrm{~W}$ to $600 \mathrm{~W}$ ) over a variable time period $(1 \mathrm{~min}$ to $1 \mathrm{~h}$ ) and the starting material was quantitatively recovered. The use of basic alumina or Montmorillonite $\mathrm{K} 10$ as solid supports and raising the irradiation power to $1600 \mathrm{~W}$ was equally ineffective. 
Addition of base ( $N$-methylpiperazine or aqueous sodium carbonate) also resulted in the recovery of the starting material.

The substitution pattern of these compounds associated with their high stability makes them convenient compounds to be tested for their antipsychotic activity and also to be used as ligands for the preparation of metal complex catalysts.

\section{Experimental Section}

General. All compounds were fully characterized by elemental analysis and spectroscopic data. The NMR spectra were recorded on a Varian Unity Plus $\left({ }^{1} \mathrm{H}: 300 \mathrm{MHz},{ }^{13} \mathrm{C}: 75 \mathrm{MHz}\right)$, or Bruker Avance $\mathrm{II}^{+} 400\left({ }^{1} \mathrm{H}: 400 \mathrm{MHz},{ }^{13} \mathrm{C}: 100 \mathrm{MHz}\right.$ ) including the ${ }^{1} \mathrm{H}-{ }^{13} \mathrm{C}$ correlation spectra (HMQC and $\mathrm{HMBC}$ ) and deuterated DMSO was used as solvent. IR spectra were recorded on a FT-IR Bomem MB 104 using Nujol mulls and $\mathrm{NaCl}$ cells. The reactions were monitored by thin layer chromatography (TLC) using silica gel 60 F254 (Merck). The melting points were determined on a Stuart SMP3 melting point apparatus and are uncorrected. Elemental analyses were performed on a LECO CHNS-932 instrument.

\section{Representative procedure for the reaction of $o$-phenylenediamine with ethoxymethylene} compounds

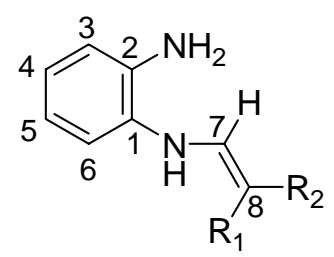

2-((2-Aminophenylamino)methylene)malononitrile (8a). Ethoxymethylene- malononitrile 7a ( $0.46 \mathrm{~g} ; 3.73 \mathrm{mmol})$ was added to a solution of $o$-phenylenediamine $1(0.40 \mathrm{~g} ; 3.73 \mathrm{mmol})$ in ethanol $(3 \mathrm{~mL})$. After $2 \mathrm{~min}$. at room temperature, a yellow solid started to precipitate and was filtered and washed with water. The product was identified as 2-((2aminophenylamino)methylene)malononitrile 8a (0.39 g; $2.10 \mathrm{mmol} ; 56 \%)$ : M.p. 107-109 ${ }^{\circ} \mathrm{C}$; IR (Nujol mull) 3436, 3361, 3266, 3224, 2221, 2206, 1652, 1635, 1506, 1461, 1377, 1344, 1318, $1303,1288,1220,1187,1143 \mathrm{~cm}^{-1}$; The NMR spectrum shows a 1:3.5 mixture of isomers A and B; ${ }^{1} \mathrm{H}-\mathrm{NMR}\left(300 \mathrm{MHz}, \mathrm{DMSO}-\mathrm{d}_{6}\right) 5.17$ (brs, 2H, NH (A and B)), 6.49 (dt, $1 \mathrm{H}, H-5(\mathrm{~A}), J_{1} 1.2$ $\mathrm{Hz}$ and $\left.J_{2} 7.5 \mathrm{~Hz}\right), 6.58\left(\mathrm{dt}, 1 \mathrm{H}, H-5(\mathrm{~B}), J_{1} 1.2 \mathrm{~Hz}\right.$ and $\left.J_{2} 7.5 \mathrm{~Hz}\right), 6.67$ (d, 1H, H-3(A), J 7.2 Hz), $6.73\left(\mathrm{dd}, 1 \mathrm{H}, H-3(\mathrm{~B}), J_{1} 1.2 \mathrm{~Hz}\right.$ and $\left.J_{2} 7.8 \mathrm{~Hz}\right), 6.91(\mathrm{~d}, 1 \mathrm{H}, H-4(\mathrm{~A}), J 7.5 \mathrm{~Hz}), 6.99(\mathrm{dt}, 1 \mathrm{H}, H-$ 4(B), $J_{1} 1.2 \mathrm{~Hz}$ and $\left.J_{2} 7.8 \mathrm{~Hz}\right), 7.05\left(\mathrm{dd}, 1 \mathrm{H}, H-6(\mathrm{~B}), J_{1} 1.2 \mathrm{~Hz}\right.$ and $\left.J_{2} 7.8 \mathrm{~Hz}\right), 7.72(\mathrm{~d}, 1 \mathrm{H}, H-$ 7(A), J 7.2 Hz), 8.04 (s, 1H, H-7(B)), 10.20 (d, 1H, N-H(A), J 7.2 Hz)), 11.0-9.0 (brs, 1H, N$\mathrm{H}(\mathrm{B})) ;{ }^{13} \mathrm{C}-\mathrm{NMR}\left(75 \mathrm{MHz}, \mathrm{DMSO}-\mathrm{d}_{6}\right) 48.79$ (C8(A)), 50.64 (C8(B)), 112.10 (CN(B)), 113.3 
(CN(A)), 114.60 (CN(B)), 114.94 (C3(A)), 115.27 (C5(A)), 116.15 (C3(B)), 116.76 (C5(B)), 119.55 (C1(A)), 123.56 (C6(B)), 125.52 (C1(B)), 127.68 (C4(A and B)), 129.48 (C6(A)), 141.66 (C2(B)), $144.70(\mathrm{C} 2(\mathrm{~A})), 157.30(\mathrm{CH}(\mathrm{A})), 159.40(\mathrm{CH}(\mathrm{B}))$; Anal. Calcd. for $\mathrm{C}_{10} \mathrm{H}_{8} \mathrm{~N}_{4} \cdot 0.13 \mathrm{H}_{2} \mathrm{O}$ : C, 64.38; H, 4.47; N, $30.04 \%$. Found: C, 64.42; H, 4.47; N, $29.95 \%$.

Ethyl 3-(2-aminophenylamino)-2-cyanoacrylate (8b). 89 \%; M.p. $154-156{ }^{\circ} \mathrm{C}$; IR (Nujol mull) $3400,3343,2215,1666,1646,1619,1584,1504,1462,1417,1379,1352,1335,1312,1280$, $1251,1174,1157,1964,1025,1004 \mathrm{~cm}^{-1}$; The NMR spectrum shows a 1.1:0.9 mixture of isomers $\mathrm{A}$ and $\mathrm{B} ;{ }^{1} \mathrm{H}-\mathrm{NMR}\left(400 \mathrm{MHz}, \mathrm{DMSO}-\mathrm{d}_{6}\right) 1.28$ (t, $3 \mathrm{H}, \mathrm{CH}_{3}(\mathrm{~A}$ and $\mathrm{B})$ ), $4.16\left(\mathrm{q}, 2 \mathrm{H}, \mathrm{CH}_{2}\right.$, $J 7.2 \mathrm{~Hz}(\mathrm{~B})), 4.23$ (q, 2H, CH,$J 7.2 \mathrm{~Hz}(\mathrm{~A})), 5.02$ (brs, $2 \mathrm{H}, \mathrm{NH}_{2}$ (A and B)), 6.61 (dt, $1 \mathrm{H}, \mathrm{H}-$ 5(B), $J_{1} 1.2 \mathrm{~Hz}$ and $\left.J_{2} 7.6 \mathrm{~Hz}\right), 6.70\left(\mathrm{dt}, 1 \mathrm{H}, H-5(\mathrm{~A}), J_{1} 1.2 \mathrm{~Hz}\right.$ and $\left.J_{2} 7.6 \mathrm{~Hz}\right), 6.76(\mathrm{dd}, 1 \mathrm{H}, H-$ 3(B), $J_{1} 0.8 \mathrm{~Hz}$ and $\left.J_{2} 8.0 \mathrm{~Hz}\right), 6.85\left(\mathrm{dd}, 1 \mathrm{H}, H-3(\mathrm{~A}), J_{1} 1.2 \mathrm{~Hz}\right.$ and $\left.J_{2} 8.0 \mathrm{~Hz}\right), 6.96(\mathrm{dt}, 1 \mathrm{H}, \mathrm{H}-$ 4(A), $J_{1} 1.2 \mathrm{~Hz}$ and $J_{2} 8.0 \mathrm{~Hz}$ ), 6.99-7.04 (m, 2H, $H-4$ and $H-6(\mathrm{~B})$ ), 7.29 (dd, $1 \mathrm{H}, H-6(\mathrm{~A}), J_{1} 1.2$ $\mathrm{Hz}$ and $J_{2} 8.0 \mathrm{~Hz}$ ), 7.94 (brs, $1 \mathrm{H}, \mathrm{H}-7(\mathrm{~B})$ ), 8.31 (d, 1H, $H-7(\mathrm{~A}), J 13.6 \mathrm{~Hz}$ ), 9.95 (brs, $1 \mathrm{H}, N$ $\mathrm{H}(\mathrm{B})), 10.49$ (d, 1H, N-H(A), J 14.0 Hz); ${ }^{13} \mathrm{C}-\mathrm{NMR}\left(100 \mathrm{MHz}, \mathrm{DMSO}-\mathrm{d}_{6}\right) 14.27\left(\mathrm{CH}_{3}(\mathrm{~B})\right.$ ), $14.34\left(\mathrm{CH}_{3}(\mathrm{~A})\right), 60.09\left(\mathrm{CH}_{2}(\mathrm{~B})\right), 60.26\left(\mathrm{CH}_{2}(\mathrm{~A})\right), 72.66(\mathrm{C} 8(\mathrm{~A})), 73.44(\mathrm{C} 8(\mathrm{~B})), 116.17$ (CN(B)), 116.24 (C3(B)), 116.96 (C5(A)), 117.52 (C3(A)), 118.24 (C5(A)), 118.39 (CN(A)), 118.92 (C6(A)), 123.16 (C6(B)), 126.16 (C1(B), 126.37 (C4(A)), 126.69 (C1(A)), 127.43 (C4(B)), 139.59 (C2(A)), 141.65 (C2(B)), 154.56 (CH(A)), 156.37 (CH(B)), $164.84(\mathrm{CO}(\mathrm{B}))$, $166.76(\mathrm{CO}(\mathrm{A}))$; Anal. Calcd. for $\mathrm{C}_{12} \mathrm{H}_{13} \mathrm{~N}_{3} \mathrm{O}_{2}$ : C, 62.31; H, 5.68; N, 18.17\%. Found: C, 62.33; H, 5.83; N, 17.96\%.

Diethyl 2-((2-aminophenylamino)methylene)malonate (8c). 89 \%; M.p. 94-96 ${ }^{\circ} \mathrm{C}$; IR (Nujol mull) 3409, 3350, 3247, 1698, 1655, 1609, 1593, 1501, 1463, 1414, 1377, 1365, 1347, 1326, 1312, 1293, 1249, 1223, 1168, 1152, 1099, 1069, 1029, $1001 \mathrm{~cm}^{-1}$; ${ }^{1} \mathrm{H}-\mathrm{NMR}$ (300 MHz, DMSOd 6$) 1.23\left(\mathrm{t}, 3 \mathrm{H}, \mathrm{CH}_{3}, J 7.2 \mathrm{~Hz}\right), 1.25$ (t, 3H, CH,$\left.J 6.9 \mathrm{~Hz}\right), 4.09$ (q, 2H, CH,$\left.J 7.2 \mathrm{~Hz}\right), 4.18$ (q, $\left.2 \mathrm{H}, \mathrm{CH}_{2}, J 6.9 \mathrm{~Hz}\right), 4.99\left(\mathrm{~s}, 2 \mathrm{H}, \mathrm{NH}_{2}\right), 6.69\left(\mathrm{dt}, 1 \mathrm{H}, H-5, J_{1} 1.5 \mathrm{~Hz}\right.$ and $\left.J_{2} 7.7 \mathrm{~Hz}\right), 6.83(\mathrm{dd}, 1 \mathrm{H}$, $H-3, J_{1} 1.5 \mathrm{~Hz}$ and $\left.J_{2} 8.1 \mathrm{~Hz}\right), 6.96\left(\mathrm{dt}, 1 \mathrm{H}, H-4, J_{1} 1.5 \mathrm{~Hz}\right.$ and $\left.J_{2} 7.7 \mathrm{~Hz}\right), 7.13\left(\mathrm{dd}, 1 \mathrm{H}, H-6, J_{1}\right.$ $1.2 \mathrm{~Hz}$ and $\left.J_{2} 7.8 \mathrm{~Hz}\right), 8.25$ (d, $\left.1 \mathrm{H}, \mathrm{H}-7, J 13.8 \mathrm{~Hz}\right), 10.43(\mathrm{~d}, 1 \mathrm{H}, \mathrm{N}-\mathrm{H}, J 14.1 \mathrm{~Hz}) ;{ }^{13} \mathrm{C}-\mathrm{NMR}(75$ MHz, DMSO-d 6$) 14.25\left(\mathrm{CH}_{3}\right), 14.30\left(\mathrm{CH}_{3}\right), 59.20\left(\mathrm{CH}_{2}\right), 59.41\left(\mathrm{CH}_{2}\right), 92.23(\mathrm{C} 8), 117.25(\mathrm{C} 3)$, 118.20 (C5), 118.93 (C6), 126.12 (C4), 127.21 (C1), 139.94 (C2), 153.18 (CH), 164.95 (CO), 167.55 (CO); Anal. Calcd. for $\mathrm{C}_{14} \mathrm{H}_{18} \mathrm{~N}_{2} \mathrm{O}_{4}$ : C, 60.41; H, 6.53; N, 10.07\%. Found: C, 60.18; H, $6.46 ; \mathrm{N}, 10.21 \%$.

\section{Representative procedure for the reaction of compounds 8a-c with aromatic aldehydes}

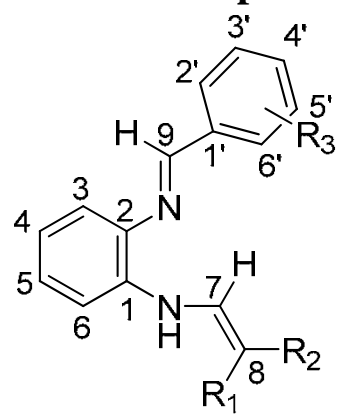


Ethyl 2-cyano-3-(2-(4-hydroxybenzylideneamino)phenylamino)acrylate (10b). Piperidine (1 drop) was added to a solution of ethyl 3-(2-aminophenylamino)-2-cyanoacrylate $\mathbf{8 b}$ ( $0.09 \mathrm{~g} ; 0.41$ $\mathrm{mmol})$ and 4-hydroxybenzaldehyde $(0.05 \mathrm{~g}$; $0.41 \mathrm{mmol})$ in acetonitrile $(1 \mathrm{~mL})$. The mixture was stirred at room temperature and after $1.5 \mathrm{~h}$, a yellow solid precipitated from solution and was filtered and washed with a 1:1 mixture of cold diethyl ether and petroleum ether. The product was identified as ethyl 2-cyano-3-(2-(4-hydroxybenzylideneamino)phenylamino)acrylate 10b (0.13 g; 0.39 mmol; 95\%): M.p. 144-147 ${ }^{\circ} \mathrm{C}$; IR (Nujol mull) 3183, 2207, 1681, 1617, 1604, 1589, 1575, 1551, 1501 1461, 1377, 1353, 1315, 1294, 1242, 1208, 1242, 1208, 1164, 1099, $1029 \mathrm{~cm}^{-1}$; ${ }^{1} \mathrm{H}-\mathrm{NMR}\left(300 \mathrm{MHz}, \mathrm{DMSO}-\mathrm{d}_{6}\right) 1.27$ (t, 3H, $\mathrm{CH}_{3}, J 7.2 \mathrm{~Hz}$ ), 4.26 (q, 2H, CH,$J 7.2$ $\mathrm{Hz}), 6.88$ (d, 2H, H-3' and $H-5$ ', J $8.7 \mathrm{~Hz}), 7.17$ (t, 1H, H-4, J 7.5 Hz), $7.26(\mathrm{t}, 1 \mathrm{H}, H-5, J 7.5$ Hz), 7.47 (d, 1H, H-3, J 7.2 Hz), 7.74 (d, 1H, H-6, J 8.1 Hz), 7.96 (d, 2H, H-2' and H-6', J 8.4 $\mathrm{Hz}), 8.67$ (s, 1H, H-7), 8.71 (s, 1H, H-9); ${ }^{13} \mathrm{C}-\mathrm{NMR}\left(75 \mathrm{MHz}, \mathrm{DMSO}-\mathrm{d}_{6}\right) 14.20\left(\mathrm{CH}_{3}\right), 60.46$ $\left(\mathrm{CH}_{2}\right), 73.75(\mathrm{C} 8), 114.31(\mathrm{C} 6), 115.86\left(\mathrm{C}^{\prime}\right.$ ' and 5'), $117.47(\mathrm{C} 3), 118.26(\mathrm{CN}), 125.11(\mathrm{C} 4)$, 126.97 (C1'), 127.10 (C5), 131.41 (C2' and 6'), 133.14 (C1), 138.45 (C2), 151.33 (C7), 159.70 (CH), 161.90 (C4'), 166.69 (CO); Anal. Calcd. for $\mathrm{C}_{19} \mathrm{H}_{17} \mathrm{~N}_{3} \mathrm{O}_{3} .0 .3 \mathrm{C}_{5} \mathrm{H}_{11} \mathrm{~N} .0 .25 \mathrm{H}_{2} \mathrm{O}$ : C, 67.39; H, 5.70; N, 12.66\%. Found: C, 67.07; H, 6.07; N, 13.06\%.

2-((2-(2-Hydroxy-3-methoxybenzylideneamino)phenylamino)methylene)malononitrile (10a). 48 \%; M.p. 164-166 ${ }^{\circ} \mathrm{C}$; IR (Nujol mull) 3205, 2221, 2202, 1636, 1614, 1588, 1573, 1503, 1462 , 1381, 1360, 1317, 1276, 1251, 1187, 1189, 1108, 1096, $1077 \mathrm{~cm}^{-1}$; ${ }^{1} \mathrm{H}-\mathrm{NMR}$ (400 MHz, DMSOd $\mathrm{d}_{6} 3.83\left(\mathrm{~s}, 3 \mathrm{H}, \mathrm{OCH}_{3}\right), 6.92(\mathrm{t}, 1 \mathrm{H}, \mathrm{H}-5$ ', J $8.0 \mathrm{~Hz}), 7.15$ (dd, $1 \mathrm{H}, \mathrm{H}-4$ ', $J_{1} 1.2 \mathrm{~Hz}$ and $\left.J_{2} 8.0 \mathrm{~Hz}\right)$, 7.3-7.4 (m, 3H, H-4, H-5 and H-6'), 7.4-7.5 (m, 2H, H-3 and $H-6$ ), 8.35 (brs, $1 \mathrm{H}, H-7$ ), 8.93 (brs, $1 \mathrm{H}, \mathrm{H}-9), 10.61$ (brs, $1 \mathrm{H}, \mathrm{OH}), 11.89$ (s, 1H, N-H); ${ }^{13} \mathrm{C}-\mathrm{NMR}\left(100 \mathrm{MHz}, \mathrm{DMSO}-\mathrm{d}_{6}\right) 51.53$ (C8), $55.95\left(\mathrm{OCH}_{3}\right), 114.06(\mathrm{CN}), 115.80\left(\mathrm{C}^{\prime}\right), 116.11(\mathrm{CN}), 118.85(\mathrm{C} 5$ '), $119.46(\mathrm{C} 3), 120.13$ (C1'), 121.80 (C6), 122.83 (C6'), 127.24 (C4), 127.52 (C5), 133.30 (C1), 141.00 (C2), 147.99 (C3'), 149.96 (C2'), $158.73(\mathrm{CH}), 163.19$ (imine $\mathrm{CH}$ ); Anal. Calcd. for $\mathrm{C}_{18} \mathrm{H}_{14} \mathrm{~N}_{4} \mathrm{O}_{2}$ : C, 67.90; $\mathrm{H}$, 4.44; N, 17.60\%. Found: C, 67.53; H, 4.56; N, 17.77\%.

Ethyl 2-cyano-3-(2-(4-methoxybenzylideneamino)phenylamino)acrylate (10c). 83 \%; M.p. 205-208 ${ }^{\circ} \mathrm{C}$; IR (Nujol mull) 3200, 2207, 1679, 1614, 1604, 1585, 1569, 1513, 1496, 1462, 1424, 1376i, 1351, 1310, 1246, 1171, $1025 \mathrm{~cm}^{-1}$; ${ }^{1} \mathrm{H}-\mathrm{NMR}$ (300 MHz, DMSO-d 6$) 1.29$ (t, 3H, $\mathrm{CH}_{3}, J$ $8.7 \mathrm{~Hz}$ ), 4.28 (q, 2H, CH,$J 6.9 \mathrm{~Hz}), 3.86$ (s, $\left.1 \mathrm{H}, \mathrm{OCH}_{3}\right), 7.09$ (d, 2H, H-3' and H-5', J 6.9 Hz), $7.19\left(\mathrm{dt}, 1 \mathrm{H}, H-4, J_{1} 1.2 \mathrm{~Hz}\right.$ and $\left.J_{2} 7.8 \mathrm{~Hz}\right), 7.29\left(\mathrm{dt}, 1 \mathrm{H}, H-5, J_{1} 1.2 \mathrm{~Hz}\right.$ and $\left.J_{2} 7.8 \mathrm{~Hz}\right), 7.48$ (dd, $1 \mathrm{H}, H-3, J_{1} 1.2 \mathrm{~Hz}$ and $\left.J_{2} 7.8 \mathrm{~Hz}\right), 7.74\left(\mathrm{dd}, 1 \mathrm{H}, H-6, J_{1} 1.2 \mathrm{~Hz}\right.$ and $\left.J_{2} 8.1 \mathrm{~Hz}\right), 8.08(\mathrm{~d}, 2 \mathrm{H}, H-2$ ' e $H-6$ ', J 8.7 Hz), 8.60 (d, 1H, H-7, J $14.4 \mathrm{~Hz}), 8.77$ (s, 1H, H-9), 11.77 (d, 1H, N-H, J 14.1 Hz); ${ }^{13} \mathrm{C}-\mathrm{NMR}$ (75 MHz, DMSO-d6) $13.90\left(\mathrm{CH}_{3}\right), 55.26\left(\mathrm{OCH}_{3}\right), 60.19\left(\mathrm{CH}_{2}\right), 73.90(\mathrm{C} 8), 114.13$ (C3' and 5'), 114.25 (C6), 117.27 (C3), $117.73(\mathrm{CN}), 124.83$ (C4), 127.11 (C5), 128.63 (C1'), 130.83 (C2' and 6'), $133.04(\mathrm{C} 1), 138.19$ (C2), $151.07(\mathrm{CH}), 159.32$ (imine $\mathrm{CH}), 162.26$ (C4'), 166.37 (CO); Anal. Calcd. for $\mathrm{C}_{20} \mathrm{H}_{19} \mathrm{~N}_{3} \mathrm{O}_{3}$ : C, 68.74; H, 5.49; N, 12.03\%. Found: C, 68.59; H, $5.48 ; \mathrm{N}, 12.15 \%$.

Ethyl 3-(2-(4-chlorobenzylideneamino)phenylamino)-2-cyanoacrylate (10d). 81 \%; M.p. 185-188 ${ }^{\circ} \mathrm{C}$; IR (Nujol mull) 3181, 2212, 1672, 1614, 1591, 1578, 1500, 1485, 1463, 1419, 1396, 
1378, 1354, 1315, 1248, 1168, 1155, 1088, 1046, 1027, $1011 \mathrm{~cm}^{-1} ;{ }^{1} \mathrm{H}-\mathrm{NMR}$ (300 MHz, DMSOd6) 1.27 (t, 3H, $\left.\mathrm{CH}_{3}, J 7.2 \mathrm{~Hz}\right), 4.26$ (q, 2H, CH,$\left.J 7.2 \mathrm{~Hz}\right), 7.20$ (t, 1H, H-4, J 6.9 Hz), 7.32 (t, $1 \mathrm{H}, H-5, J 7.2 \mathrm{~Hz}), 7.54$ (d, $1 \mathrm{H}, H-3, J 6.9 \mathrm{~Hz}), 7.59$ (d, 2H, H-3' and $H-5$ ', J $8.4 \mathrm{~Hz}), 7.74$ (d, $1 \mathrm{H}, H-6, J 8.1 \mathrm{~Hz}$ ), 8.02 (d, 2H, H-2' and H-6', J 8.4 Hz), 8.65 (d, $1 \mathrm{H}, H-7, J 14.1 \mathrm{~Hz}), 8.85$ (s, 1H, H-9), 11.89 (d, 1H, N-H, J 14.4 Hz); ${ }^{13} \mathrm{C}-\mathrm{NMR}\left(75 \mathrm{MHz}, \mathrm{DMSO}-\mathrm{d}_{6}\right) 14.25\left(\mathrm{CH}_{3}\right), 60.66$ $\left(\mathrm{CH}_{2}\right), 74.12$ (C8), 114.51 (C6), 117.70 (C3), $118.21(\mathrm{CN}), 125.11$ (C4), 128.42 (C5), 129.05 (C3' and 5'), 130.80 (C2' and 6'), 133.65 (C1), 136.68 (C4'), 134.78 (C1'), 137.34 (C2), 151.45 $(\mathrm{CH}), 158.88$ (imine $\mathrm{CH}$ ), $166.84(\mathrm{CO})$; Anal. Calcd. for $\mathrm{C}_{19} \mathrm{H}_{16} \mathrm{ClN}_{3} \mathrm{O}_{2}$ : C, 64.49; $\mathrm{H}, 4.57$; , $11.88 \%$. Found: C, 64.47; H, 4.81; N, 12.02\%.

Ethyl 2-cyano-3-(2-(4-cyanobenzylideneamino)phenylamino)acrylate (10e). 89 \%; M.p. 244$247{ }^{\circ} \mathrm{C}$; IR (Nujol mull) 3198, 3062, 2225, 2214, 1678, 1624, 1601, 1593, 1496, 1462, 1415 , 1390, 1376, 1357, 1316, 1299, 1240, 1175, 1152, 1090, $1026 \mathrm{~cm}^{-1}$; ${ }^{1} \mathrm{H}-\mathrm{NMR}$ (400 MHz, DMSOd $) 1.28$ (t, 3H, $\left.\mathrm{CH}_{3}, J 7.2 \mathrm{~Hz}\right), 4.29$ (q, 2H, $\left.\mathrm{CH}_{2}, J 7.2 \mathrm{~Hz}\right), 7.22$ (t, 1H, H-4, J $7.6 \mathrm{~Hz}$ ), 7.37 (t, $1 \mathrm{H}, H-5, J 7.6 \mathrm{~Hz}), 7.60$ (d, 1H, H-3, J $8.0 \mathrm{~Hz}$ ), 7.78 (d, 1H, H-6, J $8.4 \mathrm{~Hz}), 8.01$ (d, 2H, H-3' and $H-5$ ', $\left.J_{1} 8.0 \mathrm{~Hz}\right), 8.28$ (d, 2H, H-2' and H-6', $\left.J_{1} 8.4 \mathrm{~Hz}\right), 8.70$ (d, $\left.1 \mathrm{H}, H-7, J 14.0 \mathrm{~Hz}\right), 8.98$ (s, 1H, H-9), 11.95 (d, 1H, N-H, J 14.0 Hz); ${ }^{13} \mathrm{C}-\mathrm{NMR}$ (100 MHz, DMSO-d $) 14.19\left(\mathrm{CH}_{3}\right), 60.62$ $\left(\mathrm{CH}_{2}\right), 74.24$ (C8), 114.60 (C6), $117.72(\mathrm{C} 3), 118.02(\mathrm{CN}), 118.48$ (C4'), 125.01 (C4), 129.01 (C5), 129.53 (C2' and 6'), 133.93 (C1), 132.77 (C3' and 5'), 136.82 (C2), 139.70 (C1'), 151.49 $(\mathrm{CH}), 158.50$ (imine $\mathrm{CH}$ ), 166.81 (CO); Anal. Calcd. for $\mathrm{C}_{20} \mathrm{H}_{16} \mathrm{~N}_{4} \mathrm{O}_{2}$ : C, 69.74; $\mathrm{H}, 4.69$; $\mathrm{N}$, $16.27 \%$. Found: C, 69.74; H, 4.81; N, 16.31\%.

Ethyl 2-cyano-3-(2-(4-nitrobenzylideneamino)phenylamino)acrylate (10f). 78 \%; M.p. 244$247{ }^{\circ} \mathrm{C}$; IR (Nujol mull) 3200, 2215, 1679, 1624, 1592, 1576, 1520, 1503, 1462, 1376, 1357 , 1338, 1315, 1240, 1176, 1152, 1108, 1090, $1024 \mathrm{~cm}^{-1} ;{ }^{1} \mathrm{H}-\mathrm{NMR}\left(300 \mathrm{MHz}, \mathrm{DMSO}-\mathrm{d}_{6}\right) 1.29(\mathrm{t}$, $3 \mathrm{H}, \mathrm{CH}_{3}, J 7.2 \mathrm{~Hz}$ ), 4.30 (q, 2H, $\mathrm{CH}_{2}, J 7.2 \mathrm{~Hz}$ ), 7.22 (t, 1H, H-4, J 7.2 Hz), 7.37 (t, 1H, H-5, J $7.2 \mathrm{~Hz}$ ), 7.61 (d, 1H, H-3, J 7.2 Hz), 7.78 (d, 1H, H-6, J 7.8 Hz), 8.35 (s, 4H, H-3', H-5', H-2' and $H-6$ '), 8.70 (d, 1H, H-7, J 14.1 Hz), 9.03 (s, 1H, H-9), 11.96 (d, $1 \mathrm{H}, \mathrm{N}-H, J 13.8 \mathrm{~Hz})$; ${ }^{13} \mathrm{C}-$ NMR (75 MHz, DMSO-d $) 14.20\left(\mathrm{CH}_{3}\right), 60.70\left(\mathrm{CH}_{2}\right), 74.32$ (C8), 114.62 (C6), 117.77 (C3), 118.05 (CN), 123.99 (C3' and 5'), 125.03 (C4), 129.24 (C5), 130.03 (C2' and 6'), 134.06 (C1), 136.73 (C2), 141.38 (C1'), 149.04 (C4'), 151.49 (CH), 158.04 (imine $\mathrm{CH}), 166.85$ (CO); Anal. Calcd. for $\mathrm{C}_{19} \mathrm{H}_{16} \mathrm{~N}_{4} \mathrm{O}_{4}$ : C, 62.62; H, 4.44; N, 15.38\%. Found: C, 62.51; H, 4.65; N, 15.43\%.

Ethyl 2-cyano-3-(2-(2-hydroxy-3-methoxybenzylideneamino)phenylamino)acrylate (10g). 80 \%; M.p. 146-148 ${ }^{\circ} \mathrm{C}$; IR (Nujol mull) 2202, 1681, 1625, 1612, 1593, 1573, 1500, 1461, 1444, $1416,1395,1377,1355,1313,1278,1260,1245,1214,1177,1150,1102,1078,1025,1050 \mathrm{~cm}^{-}$

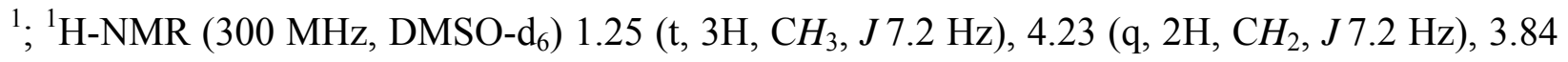
$\left(\mathrm{s}, 3 \mathrm{H}, \mathrm{OCH}_{3}\right), 6.91\left(\mathrm{dt}, 1 \mathrm{H}, \mathrm{H}_{-} 5^{\prime}, J_{1} 1.8 \mathrm{~Hz}\right.$ and $\left.J_{2} 7.8 \mathrm{~Hz}\right), 7.14\left(\mathrm{dd}, 1 \mathrm{H}, H-4^{\prime}, J_{1} 1.2 \mathrm{~Hz}\right.$ and $J_{2}$ $7.8 \mathrm{~Hz}), 7.23\left(\mathrm{dt}, 1 \mathrm{H}, \mathrm{H}-4, J_{1} 1.2 \mathrm{~Hz}\right.$ and $J_{2} 7.8 \mathrm{~Hz}$ ), 7.31 (dt, $1 \mathrm{H}, H-5, J_{1} 1.2 \mathrm{~Hz}$ and $J_{2} 7.8 \mathrm{~Hz}$ ), $7.43\left(\mathrm{dd}, 1 \mathrm{H}, H-3, J_{1} 1.2 \mathrm{~Hz}\right.$ and $\left.J_{2} 7.8 \mathrm{~Hz}\right), 7.68\left(\mathrm{dd}, 1 \mathrm{H}, H-6\right.$ ', $J_{1} 1.2 \mathrm{~Hz}$ and $\left.J_{2} 7.8 \mathrm{~Hz}\right), 7.75$ (dd, 1H, H-6, J 7.8 Hz), 8.65 (d, 1H, H-7, J 13.8 Hz), 9.03 (s, 1H, H-9), 10.55 (s, 1H, OH), 11.50 $(\mathrm{d}, 1 \mathrm{H}, \mathrm{N}-\mathrm{H}, \mathrm{J} 13.8 \mathrm{~Hz}) ;{ }^{13} \mathrm{C}-\mathrm{NMR}\left(75 \mathrm{MHz}, \mathrm{DMSO}-\mathrm{d}_{6}\right) 14.17\left(\mathrm{CH}_{3}\right), 55.99\left(\mathrm{OCH}_{3}\right), 60.56$ $\left(\mathrm{CH}_{2}\right), 74.18$ (C8), 115.43 (C4'), 115.24 (C6), 118.09 (CN), 118.52 (C3), 119.07 (C5'), 121.00 
(C6'), 121.38 (C1'), 125.52 (C4), 127.75 (C5), 132.89 (C1), 138.70 (C2), 148.07 (C3'), 149.06 (C2'), $152.05(\mathrm{CH}), 159.75$ (imine $\mathrm{CH}$ ), $166.60(\mathrm{CO})$; Anal. Calcd. for $\mathrm{C}_{20} \mathrm{H}_{19} \mathrm{~N}_{3} \mathrm{O}_{4}$ : C, 65.73; $\mathrm{H}$, 5.25; N, 11.50\%. Found: C, 65.56; H, 5.30; N, 11.62\%.

Diethyl 2-((2-(4-hydroxybenzylideneamino)phenylamino)methylene)malonate (10h). $55 \%$; M.p. 183-186 ${ }^{\circ}$; IR (Nujol mull) 3190, 1675, 1656, 1629, 1608, 1590, 1571, 1519, 1495, 1462 , 1416, 1378, 1366, 1352, 1324, 1282, 1251, 1220, 1165, 1113, 1092, 1043, $1032 \mathrm{~cm}^{-1}$; ${ }^{1} \mathrm{H}-\mathrm{NMR}$ (300 MHz, DMSO-d $) 1.23$ (t, 3H, $\mathrm{CH}_{3}, J 6.9 \mathrm{~Hz}$ ), 1.27 (t, 3H, $\mathrm{CH}_{3}, J 7.2 \mathrm{~Hz}$ ), 4.12 (q, 2H, $\mathrm{CH}_{2}$, $J 6.9 \mathrm{~Hz}), 4.23$ (q, 2H, $\left.\mathrm{CH}_{2}, J 7.2 \mathrm{~Hz}\right), 6.90$ (d, 2H, H-3' and H-5', J 8.7 Hz), 7.15 (t, $1 \mathrm{H}, H-4, J$ $6.9 \mathrm{~Hz}), 7.27$ (t, 1H, H-5, J 7.2 Hz), 7.44 (d, 1H, H-3, J6.9 Hz), 7.55 (d, 1H, H-6, J 7.8 Hz), 7.97 (d, 2H, H-2' and H-6', J 8.7 Hz), 8.57 (d, 1H, H-7, J 14.1 Hz), 8.70 (s, 1H, H-9), $11.78(\mathrm{~d}, 1 \mathrm{H}$, N-H, J 14.1 Hz); ${ }^{13} \mathrm{C}-\mathrm{NMR}\left(75 \mathrm{MHz}, \mathrm{DMSO}-\mathrm{d}_{6}\right) 14.22\left(\mathrm{CH}_{3}\right), 14.26\left(\mathrm{CH}_{3}\right), 55.43\left(\mathrm{CH}_{2}\right), 59.58$ $\left(\mathrm{CH}_{2}\right), 93.35$ (C8), 113.92 (C6), 115.75 (C3' and 5'), 117.63 (C3), 124.55 (C4), 126.97 (C1'), 127.28 (C5) 131.33 (C2' and 6'), 133.71 (C1), 138.68 (C2), 149.19 (CH), 159.54 (imine $\mathrm{CH}$ ), 161.39 (C4'), 164.93 (CO), 167.65 (CO); Anal. Calcd. for $\mathrm{C}_{21} \mathrm{H}_{22} \mathrm{~N}_{2} \mathrm{O}_{5}$ : C, 65.95; H, 5.81; N, 7.33\%. Found: C, 65.66; H, 5.95; N, 7.48\%.

Diethyl 2-((2-(4-methoxybenzylideneamino)phenylamino)methylene)malonate (10i). $74 \%$; M.p. $88-90{ }^{\circ} \mathrm{C}$; IR (Nujol mull) 1685, 1646, 1625, 1605i, 1583, 1565, 1513, 1460, 1423, 1377 , 1343, 1306, 1249, 1183, 1170, 1091, $1027 \mathrm{~cm}^{-1}$; ${ }^{1} \mathrm{H}-\mathrm{NMR}\left(300 \mathrm{MHz}, \mathrm{DMSO}-\mathrm{d}_{6}\right) 1.24(\mathrm{t}, 3 \mathrm{H}$, $\left.\mathrm{CH}_{3}, J 6.9 \mathrm{~Hz}\right), 1.27$ (t, 3H, $\left.\mathrm{CH}_{3}, J 6.9 \mathrm{~Hz}\right), 4.13$ (q, 2H, $\left.\mathrm{CH}_{2}, J 6.9 \mathrm{~Hz}\right), 4.24$ (q, 2H, $\mathrm{CH}_{2}, J 6.9$ $\mathrm{Hz}), 3.85\left(\mathrm{~s}, 3 \mathrm{H}, \mathrm{OCH}_{3}\right), 7.09\left(\mathrm{~d}, 2 \mathrm{H}, H-3\right.$ ' and $H-5$ ', J 8.7 Hz), $7.16\left(\mathrm{dt}, 1 \mathrm{H}, \mathrm{H}-4, J_{1} 1.2 \mathrm{~Hz}\right.$ and $\left.J_{2} 8.1 \mathrm{~Hz}\right), 7.30\left(\mathrm{dt}, 1 \mathrm{H}, H-5, J_{1} 1.2 \mathrm{~Hz}\right.$ and $\left.J_{2} 7.5 \mathrm{~Hz}\right), 7.48\left(\mathrm{dd}, 1 \mathrm{H}, H-3, J_{1} 1.2 \mathrm{~Hz}\right.$ and $J_{2} 7.8$ $\mathrm{Hz}), 7.56$ (d, 1H, H-6, J 7.5 Hz), 8.08 (d, 2H, H-2' and H-6', $J_{1} 1.8 \mathrm{~Hz}$ and $\left.J_{2} 6.9 \mathrm{~Hz}\right), 8.57$ (d, $1 \mathrm{H}, H-7, J 14.4 \mathrm{~Hz}$ ), 8.77 (s, $1 \mathrm{H}, H-9), 11.79$ (d, 1H, N-H, J $14.4 \mathrm{~Hz}) ;{ }^{13} \mathrm{C}-\mathrm{NMR}(75 \mathrm{MHz}$, DMSO-d6) $14.22\left(\mathrm{CH}_{3}\right), 14.23\left(\mathrm{CH}_{3}\right), 59.45\left(\mathrm{CH}_{2}\right), 59.62\left(\mathrm{CH}_{2}\right), 55.50\left(\mathrm{OCH}_{3}\right), 93.44(\mathrm{C} 8)$, 113.97 (C6), 114.34 (C3' and 5'), 117.69 (C3), 124.54 (C4), 127.58 (C5), 128.88 (C1'), 131.07 (C2' and 6'), 133.83 (C1), 138.42 (C2), 149.19 (CH), 159.41 (imine $\mathrm{CH}), 162.35$ (C4'), 164.93 (CO), 167.69 (CO); Anal. Calcd. for $\mathrm{C}_{22} \mathrm{H}_{24} \mathrm{~N}_{2} \mathrm{O}_{5}$ : C, 66.64; H, 6.11; N, 7.07\%. Found: C, 66.80; H, 6.26; N, 7.24\%.

Diethyl 2-((2-(4-chlorobenzylideneamino)phenylamino)methylene)malonate (10j). $76 \%$; M.p. $128-131{ }^{\circ} \mathrm{C}$; IR (Nujol mull) 3156, 1686, 1650, 1626, 1609, 1590, 1575, 1566, 1500, 1461, 1430, 1376, 1359, 1343, 1311, 1258, 1176, 1096, 1086, 1044, 1027, $1014 \mathrm{~cm}^{-1}$; ${ }^{1} \mathrm{H}-\mathrm{NMR}$ (400 MHz, DMSO-d 6 ) 1.24 (t, 3H, $\left.\mathrm{CH}_{3}, J 7.2 \mathrm{~Hz}\right), 1.26$ (t, 3H, $\left.\mathrm{CH}_{3}, J 7.2 \mathrm{~Hz}\right), 4.13$ (q, 2H, CH,$~ J 7.2$ $\mathrm{Hz}$ ), 4.24 (q, 2H, $\left.\mathrm{CH}_{2}, J 7.2 \mathrm{~Hz}\right), 7.18\left(\mathrm{dt}, 1 \mathrm{H}, H-4, J_{1} 0.8 \mathrm{~Hz}\right.$ and $J_{2} 8.0 \mathrm{~Hz}$ ), 7.34 (dt, $1 \mathrm{H}, H-5$, $J_{1} 0.8 \mathrm{~Hz}$ and $\left.J_{2} 7.6 \mathrm{~Hz}\right), 7.53\left(\mathrm{dd}, 1 \mathrm{H}, H-3, J_{1} 1.2 \mathrm{~Hz}\right.$ and $\left.J_{2} 8.0 \mathrm{~Hz}\right), 7.58(\mathrm{dd}, 1 \mathrm{H}, H-6, J 8.4$ $\mathrm{Hz}), 7.61$ (d, 2H, H-3' and $H-5$ ', J $8.4 \mathrm{~Hz}), 8.14$ (d, 2H, H-2' and $H-6$ ', J 8.4 Hz), 8.57 (d, $1 \mathrm{H}, H-$ 7, J 14.0 Hz), 8.88 (s, 1H, H-9), 11.85 (d, 1H, N-H, J $14.0 \mathrm{~Hz}) ;{ }^{13} \mathrm{C}-\mathrm{NMR}\left(100 \mathrm{MHz}, \mathrm{DMSO}-\mathrm{d}_{6}\right)$ $14.18\left(\mathrm{CH}_{3}\right), 14.23\left(\mathrm{CH}_{3}\right), 59.46\left(\mathrm{CH}_{2}\right), 59.69\left(\mathrm{CH}_{2}\right), 93.65(\mathrm{C} 8), 114.10(\mathrm{C} 6), 117.81(\mathrm{C} 3)$, 124.47 (C4), 128.43 (C5), 128.98 (C3' and 5'), 130.70 (C2' and 6'), 134.14 (C1), 134.85 (C1'), 136.81 (C4'), 137.61 (C2), $149.21(\mathrm{CH}), 158.76$ (imine $\mathrm{CH}), 164.85$ (CO), 167.79 (CO); Anal. Calcd. for $\mathrm{C}_{21} \mathrm{H}_{21} \mathrm{ClN}_{2} \mathrm{O}_{4}$ : C, 62.92; H, 5.29; N, 6.99\%. Found: C, 62.70; H, 5.49; N, 7.15\%. 
Diethyl 2-((2-(4-cyanobenzylideneamino)phenylamino)methylene)malonate (10k). $81 \%$; M.p. $172-175^{\circ} \mathrm{C}$; IR (Nujol mull) 2225, 1683, 1648, 1627, 1610, 1587, 1573, 1461, 1431, 1374, 1359, 1342, 1308, 1261, 1184, 1114, 1096, $1039 \mathrm{~cm}^{-1} ;{ }^{1} \mathrm{H}-\mathrm{NMR}\left(300 \mathrm{MHz}, \mathrm{DMSO}-\mathrm{d}_{6}\right) 1.24(\mathrm{t}$, $3 \mathrm{H}, \mathrm{CH}_{3}, J 7.2 \mathrm{~Hz}$ ), 1.27 (t, 3H, $\mathrm{CH}_{3}, J 7.2 \mathrm{~Hz}$ ), 4.13 (q, 2H, $\mathrm{CH}_{2}, J 7.2 \mathrm{~Hz}$ ), 4.25 (q, 2H, $\mathrm{CH}_{2}, J$ $7.2 \mathrm{~Hz}), 7.19\left(\mathrm{dt}, 1 \mathrm{H}, \mathrm{H}-4, J_{1} 1.2 \mathrm{~Hz}\right.$ and $\left.J_{2} 7.8 \mathrm{~Hz}\right), 7.37(\mathrm{t}, 1 \mathrm{H}, H-5, J 7.2 \mathrm{~Hz}), 7.58(\mathrm{dd}, 2 \mathrm{H}, H-$ 3 and $H-6, J_{1} 1.2 \mathrm{~Hz}$ and $\left.J_{2} 7.8 \mathrm{~Hz}\right), 8.02$ (d, 2H, H-3' and $H-5$ ', $\left.J 8.4 \mathrm{~Hz}\right), 8.29$ (d, 2H, H-2' and H-6', J 8.4 Hz), 8.58 (d, 1H, H-7, J 14.1 Hz), 8.97 (s, 1H, H-9), 11.93 (d, 1H, N-H, J 14.1 Hz); ${ }^{13} \mathrm{C}-\mathrm{NMR}\left(75 \mathrm{MHz}, \mathrm{DMSO}-\mathrm{d}_{6}\right) 14.20\left(\mathrm{CH}_{3}\right), 14.25\left(\mathrm{CH}_{3}\right), 59.51\left(\mathrm{CH}_{2}\right), 59.77\left(\mathrm{CH}_{2}\right), 93.81$ (C8), 113.59 (C4'), 114.22 (C6), 117.85 (C3), 118.54 (CN), 124.45 (C4), 129.13 (C5), 129.53 (C2' and 6'), 132.79 (C3' and 5'), 134.51 (C1), 137.05 (C2), 139.85 (C1'), 149.23 (CH), 158.31 (imine $\mathrm{CH}$ ), $164.83(\mathrm{CO}), 167.89(\mathrm{CO})$; Anal. Calcd. for $\mathrm{C}_{22} \mathrm{H}_{21} \mathrm{~N}_{3} \mathrm{O}_{4}: \mathrm{C}, 67.50 ; \mathrm{H}, 5.42 ; \mathrm{N}$, 10.74\%. Found: C, 67.28; H, 5.61; N, 10.86\%.

Diethyl 2-((2-(4-nitrobenzylideneamino)phenylamino)methylene)malonate (101). 61 \%; M.p. 177-179 ${ }^{\circ} \mathrm{C}$; IR (Nujol mull) 1685, 1650, 1611, 1589, 1573, 1523, 1514, 1462, 1432, 1377, 1348, 1311, 1266, 1247, 1181, 1097, $1036 \mathrm{~cm}^{-1} ;{ }^{1} \mathrm{H}-\mathrm{NMR}\left(300 \mathrm{MHz}, \mathrm{DMSO}-\mathrm{d}_{6}\right) 1.25$ (t, 3H, $\mathrm{CH}_{3}, J$ $7.2 \mathrm{~Hz}$ ), 1.29 (t, 3H, CH,$J 7.2 \mathrm{~Hz}), 4.15$ (q, 2H, $\left.\mathrm{CH}_{2}, J 7.2 \mathrm{~Hz}\right), 4.27$ (q, 2H, CH,$J 7.2 \mathrm{~Hz}$ ), $7.20(\mathrm{t}, 1 \mathrm{H}, H-4, J 7.2 \mathrm{~Hz}), 7.38(\mathrm{t}, 1 \mathrm{H}, H-5, J 7.5 \mathrm{~Hz}), 7.58(\mathrm{~d}, 2 \mathrm{H}, H-3$ and $H-6, J 8.4 \mathrm{~Hz}), 8.35$ (s, 4H, H-3', H-5', H-2' and H-6'), 8.55 (d, 1H, H-7, J $14.1 \mathrm{~Hz}), 9.01$ (s, 1H, H-9), 11.79 (d, 1H, N-H, J 14.1 Hz); ${ }^{13} \mathrm{C}-\mathrm{NMR}\left(75 \mathrm{MHz}, \mathrm{DMSO}-\mathrm{d}_{6}\right) 13.93\left(\mathrm{CH}_{3}\right), 13.88\left(\mathrm{CH}_{3}\right), 59.17\left(\mathrm{CH}_{2}\right), 59.44$ $\left(\mathrm{CH}_{2}\right), 94.05$ (C8), 114.06 (C6), 117.62 (C3), 123.58 (C3' and 5'), 124.13 (C4), 128.93 (C5), 129.71 (C2' and 6'), 134.42 (C1), 137.03 (C2), $141.27\left(\mathrm{C}^{\prime}\right.$ '), 148.85 (C4' and $\left.\mathrm{CH}\right), 157.60$ (imine $\mathrm{CH}$ ), $164.59(\mathrm{CO}), 167.52(\mathrm{CO})$; Anal. Calcd. for $\mathrm{C}_{21} \mathrm{H}_{21} \mathrm{~N}_{3} \mathrm{O}_{6}: \mathrm{C}, 61.30 ; \mathrm{H}, 5.16 ; \mathrm{N}$, $10.22 \%$. Found: C, 61.28; H, 5.35; N, 10.39\%.

Diethyl 2-((2-(2-hydroxy-3-methoxybenzylideneamino)phenylamino)methylene) malonate (10m). 93 \%; oil; IR (Nujol mull) 1713, 1693, 1659, 1652, 1614, 1590, 1574, 1500, 1462, 1377 , 1349, 1306 1255, 1186, 1168, 1076, $1029 \mathrm{~cm}^{-1}$; ${ }^{1} \mathrm{H}-\mathrm{NMR}\left(300 \mathrm{MHz}, \mathrm{DMSO}-\mathrm{d}_{6}\right) 1.21$ (t, 3H, $\left.\mathrm{CH}_{3}, J 7.2 \mathrm{~Hz}\right), 1.24$ (t, 3H, $\mathrm{CH}_{3}, J 7.2 \mathrm{~Hz}$ ), 3.84 (s, 3H, H-3', $\mathrm{OCH}_{3}$ ), 4.11 (q, 2H, $\mathrm{CH}_{2}, J 6.9$ $\mathrm{Hz}$ ), 4.19 (q, 2H, $\mathrm{CH}_{2}, J 6.9 \mathrm{~Hz}$ ), 6.91 (t, 1H, H-5', J $7.8 \mathrm{~Hz}$ ), 7.14 (dd, 1H, H-4', J $1.5 \mathrm{~Hz}$ and $\left.J_{2} 7.8 \mathrm{~Hz}\right), 7.20\left(\mathrm{dt}, 1 \mathrm{H}, \mathrm{H}-4, J_{1} 0.9 \mathrm{~Hz}\right.$ and $\left.J_{2} 7.8 \mathrm{~Hz}\right), 7.34\left(\mathrm{dt}, 1 \mathrm{H}, H-5, J_{1} 1.2 \mathrm{~Hz}\right.$ and $J_{2} 7.8$ $\mathrm{Hz}), 7.42\left(\mathrm{dd}, 1 \mathrm{H}, H-3, J_{1} 1.2 \mathrm{~Hz}\right.$ and $J_{2} 7.8 \mathrm{~Hz}$ ), $7.56\left(\mathrm{dd}, 1 \mathrm{H}, H-6 ; J_{1} 1.5 \mathrm{~Hz}\right.$ and $J_{2} 7.8 \mathrm{~Hz}$ ), $7.62\left(\mathrm{dd}, 1 \mathrm{H}, H-6\right.$ ', $J_{1} 1.5 \mathrm{~Hz}$ and $\left.J_{2} 7.8 \mathrm{~Hz}\right), 8.53$ (d, 1H, H-7, J 13,5 Hz), 9.02 (s, 1H, H-9), 11.38 (d, H, J 13.8 Hz); ${ }^{13} \mathrm{C}-\mathrm{NMR}\left(75 \mathrm{MHz}, \mathrm{DMSO}-\mathrm{d}_{6}\right) 14.18\left(\mathrm{CH}_{3}\right), 14.23\left(\mathrm{CH}_{3}\right), 59.50\left(\mathrm{CH}_{2}\right)$, $59.71\left(\mathrm{CH}_{2}\right), 56.01\left(\mathrm{OCH}_{3}\right), 93.76(\mathrm{C} 8), 115.20$ (C6), $115.51\left(\mathrm{C}^{\prime}\right), 118.82(\mathrm{C} 3), 119.04\left(\mathrm{C}^{\prime}\right)$, 121.20 (C1'), 121.49 (C6'), 125.08 (C4), 127.94 (C5), 133.46 (C1), 138.90 (C2), 148.08 (C3'), $149.22\left(\mathrm{C}^{\prime}\right.$ '), $150.03(\mathrm{CH}), 160.05$ (imine $\left.\mathrm{CH}\right), 164.85(\mathrm{CO}), 167.51(\mathrm{CO})$.

\section{Acknowledgements}

This work was supported by the University of Minho and by Fundação para a Ciência e a Tecnologia through project PPCDT/QUI/59356/2004. 


\section{References}

1. Tennant, G. In Comprehensive Organic Chemistry:The Synthesis and Reactions of Organic Compounds, Barton, D. H. R.; Ollis, W. D. Eds.; Pergamon Press: Oxford, 1979; Vol. 2, p 385.

2. Thakuria, H.; Das, G. Arkivoc 2008, 15, 321.

3. Bahrami, K.; Khodaei, M. M.; Naali, F. J. Org. Chem. 2008, 73, 6835.

4. Gogoi, P.; Konwar, D. Tetrahedron Lett. 2006, 47, 79.

5. Trivedi, R.; De, S. K.; Gibbs, R. A. J. Mol. Catal. A: Chem. 2006, 245, 8.

6. Preston, P. N. Chem. Rev. 1974, 74, 279.

7. Naeimi, H.; Salimi, F.; Rabiei, K. J. Mol. Cat. A: Chem.l 2006, 260, 100.

8. Jacob, R. G.; Dutra, L. G.; Radatz, C. S.; Mendes, S. R.; Perin, G.; Lenardão, E. J. Tetrahedron Lett. 2009, 50, 1495.

9. Varala, R.; Narseen, A.; Enugala, R.; Adapa, S. R. Tetrahedron Lett. 2007, 48, 69.

10. Perumal, S.; Mariappan, S.; Selvaraj, S. Arkivoc 2004, 8, 46.

11. Litvinov, V. P.; Yakunin, Y. Y.; Dyachenko, V. D. Chem. Het. Compounds 2001, 37, 37.

12. Salon, J.; Milata, V.; Gatial, A.; Pronayova, N.; Lesko, J.; Cernuchova, P.; Rappoport, Z.; Vo-Thanh, G.; Loupy, A. Eur. J. Org. Chem. 2005, 22, 4870.

13. Stanovnik, B.; Svete, J. Chem. Rev. 2004, 104, 2433.

14. Taylor, J. B.; Tully, W. R. J. Chem. Soc, Perkin Trans 1 1976, 1331.

15. Howe, R. K. J. Org. Chem. 1964, 34, 2983.

16. Zivec, M.; Sova, M.; Brunskole, M.; Lenarsic, R.; Rizner, T. L.; Gabec, S. J Enzyme Inhib. Med. Chem. 2007, 22, 29.

17. Santilli, A. A.; Bruce, W. F.; Osdene, T. S. J. Org. Chem. 1964, 7, 68.

18. Karplus, M. J. Am. Chem. Soc. 1963, 85, 2870.

19. Gatial, A.; Milata, V.; Zalibera, L.; Biskupic, S.; Salzer, R. J. Mol. Structure 1999, 482-483, 615.

20. Pigosova, J.; Gatial, A.; Milata, V.; Cernuchova, P.; Pronayova, N.; Liptaj, T.; Matejka, P. J. Mol. Structure 2005, 744-747, 315.

21. Polovkova, J.; Gatial, A.; Milata, V.; Cernuchova, P.; Pronayova, N.; Liptaj, T.; Matejka, P. J. Mol. Structure 2007, 829, 8.

22. Grof, M.; Gatial, A.; Milata, V.; Pronayova, N.; Summachen, L.; Salzer, R. J. Mol. Structure 2007, 843, 1.

23. Okamoto, Y.; Ueda, T. J.C.S. Chem. Comm. 1973, 11, 367.

24. Okamoto, Y.; Takagi, K. J. Het. Chem. 1987, 24, 885. 